\title{
The Higgs mass and the scale of SUSY breaking in the NMSSM
}

\author{
Lucila Zarate \\ II. Institut für Theoretische Physik der Universität Hamburg, \\ Luruper Chaussee 149, D-22761 Hamburg, Germany \\ E-mail: lucila.zarate@desy.de
}

ABSTRACT: In this letter we study the Higgs mass in the NMSSM with supersymmetry breaking at high scales $M_{\mathrm{SS}}$. With the Standard Model as the effective low energy theory, the computation of the Higgs mass relies on the matching condition of the quartic coupling $\lambda$ at $M_{\mathrm{SS}}$. In the MSSM, the latter is fixed to a semi-positive value and, thus, sets an upper bound on the SUSY-breaking scale near $M_{\mathrm{SS}} \simeq 10^{10} \mathrm{GeV}$. In the NMSSM, $\lambda\left(M_{\mathrm{SS}}\right)$ receives an additional contribution induced by the singlet which allows for negative values of $\lambda\left(M_{\mathrm{SS}}\right)$. In turn, for the measured value of the Higgs mass we find that $M_{\mathrm{SS}}$ can take any value up to the GUT scale. Furthermore, the choice of universal soft terms favors SUSY-breaking scales close to the GUT scale.

KeYWORDS: Supersymmetry Phenomenology

ARXIV EPRINT: 1601.05946 


\section{Contents}

1 Introduction 1

2 Fine-tuning a light Higgs in SUSY and matching to the Standard Model 2

3 Calculation of the Higgs mass $\quad 4$

3.1 Matching at $M_{\mathrm{SS}}$

3.2 Matching at $M_{\mathrm{GUT}} \quad 5$

4 The Higgs mass as a function of $M_{\mathrm{SS}} \quad 6$

$4.1 \lambda>0$ at SUSY-breaking scale $\quad 6$

$4.2 \lambda<0$ at SUSY-breaking scale $\quad 6$

4.3 Universal soft terms at $M_{\mathrm{GUT}} \quad 8$

$\begin{array}{lll}5 & \text { Conclusions } & 10\end{array}$

\section{Introduction}

The discovery of a Higgs boson of $126 \mathrm{GeV}$ [1-3] is consistent with low energy supersymmetry together with a small degree of fine-tuning. However the absence of supersymmetric particles in the $\mathrm{TeV}$ range questions the paradigm of naturalness as a guiding principle. If one abandons the idea of supersymmetry as a solution to the hierarchy problem, reasons remain to study realizations of supersymmetry. In particular, supersymmetry is present in string theory, which is so far the best candidate for a UV-complete theory. Generically, supersymmetric theories in four dimensions obtained from string compactifications favor supersymmetry breaking at high scales, e.g. the GUT scale [4]. ${ }^{1}$ Furthermore, present data confirmed that the SM Higgs potential becomes metastable at large energy scales [5] and supersymmetric embeddings can stabilize the EW vacuum. ${ }^{2}$

Let us consider the situation in which supersymmetry is broken at a scale $M_{\mathrm{SS}}$ such that $M_{\mathrm{EW}} \ll M_{\mathrm{SS}}$. Under no special assumptions on the corresponding pattern of soft terms, supersymmetric particles receive masses of $\mathcal{O}\left(M_{\mathrm{SS}}\right)$ and thus decouple from the low energy theory. The requirement of obtaining a light Higgs in the spectrum leads to a finetuning condition at the matching scale $M_{\mathrm{SS}}$ and leaves the Standard Model as an effective description at lower energies. However, the corresponding quartic coupling $\lambda(\mu)$ is completely fixed by the matching conditions to the supersymmetric theory at the scale $M_{\mathrm{SS}}{ }^{3}$ In turn, the latter implies that the Higgs mass is determined by the supersymmetric theory,

\footnotetext{
${ }^{1}$ Examples with low energy SUSY were constructed by special choices of the compactification data (e.g. fluxes).

${ }^{2}$ See $[6-8]$ for earlier results on the stability of the EW potential.

${ }^{3}$ Here $\mu$ denotes the energy scale.
} 
and thus, the measured value of the Higgs mass constrains the values of the parameters of the supersymmetric theory and $M_{\mathrm{SS}}$. This framework is called High-Scale Supersymmetry. A detailed study of the Higgs mass in the High-Scale Supersymmetry within the MSSM was done in [9-15]. In this case the prediction of $\lambda\left(M_{\mathrm{SS}}\right)$ is strictly positive (or zero), thus, the scale at which the quartic coupling in the SM vanishes, within uncertainties, sets an upper bound on $M_{\mathrm{SS}}$ of $M_{\mathrm{SS}} \simeq 10^{10} \mathrm{GeV}$. Hence, a GUT scale SUSY breaking in this setup is ruled out. ${ }^{4}$

In this letter we study High-Scale Supersymmetry within the singlet extension of the MSSM, i.e. Next-to-Minimal Supersymmetric Standard Model (NMSSM), for a comprehensive review see [16]. In the NMSSM the couplings of the extra singlet to the Higgs sector induce tree level corrections to the matching of $\lambda$ at the $M_{\mathrm{SS}}$ scale. Two pieces contribute to $\lambda\left(M_{\mathrm{SS}}\right)$ that are model independent and model dependent respectively. The former is strictly positive (or zero), and it can significantly modify the parameter values consistent with the Higgs mass w.r.t. the MSSM case. On the other hand, the latter contribution allows to set $\lambda\left(M_{\mathrm{SS}}\right)$ to a negative value, and thus, can extend $M_{\mathrm{SS}}$ up to the GUT or Planck scales. ${ }^{5}$ In this work we calculate the prediction for the Higgs mass in this framework and derive the respective bounds on $M_{\mathrm{SS}}$ and the NMSSM parameters.

This letter is organized as follows in section 2 we write down the matching conditions that relate the singlet-extended MSSM framework with the effective SM and discuss the fine-tuning condition required to induce a light Higgs. In section 3 we explain how we calculate the Higgs mass and in section 4 we study the dependence of the Higgs mass as a function of the SUSY-breaking scale and NMSSM parameters. In section 4.3 we study the constraints on the Higgs mass for special choices of soft parameters at the GUT scale. Finally, in section 5 we present the conclusions.

\section{Fine-tuning a light Higgs in SUSY and matching to the Standard Model}

Let us consider a scenario in which above the (not necessarily low) supersymmetry breaking scale $\left(M_{\mathrm{SS}}\right)$ the theory is described by a singlet-extended MSSM with the following superpotential

$$
W_{\mathrm{NMSSM}}=\left(\mu_{h}+y_{s} S\right) H_{u} H_{d}+\frac{1}{2} \mu_{s} S^{2}+y_{u} Q H_{u} U_{R}+y_{d} Q H_{d} D_{R}+y_{e} L H_{d} E_{R},
$$

where $S$ is the NMSSM singlet and $H_{u}, H_{d}$ are the MSSM Higgs multiplets. $y_{s}$ is a dimensionless Yukawa coupling and $\mu_{h}, \mu_{s}$ are the supersymmetric Higgs and singlet mass terms respectively. $y_{u}, y_{d}, y_{e}$ are the Yukawa couplings of the MSSM which should be understood as matrices in the family space, $Q$ are the quark doublets, $U_{R}$ and $D_{R}$ are the quark singlets, $L$ are the lepton doublets and $E_{R}$ are the lepton singlets. After supersymmetry

\footnotetext{
${ }^{4}$ This statement holds only at tree level. By introducing large one loop threshold corrections to the matching of the quartic coupling, one could fine-tune $\lambda\left(M_{\mathrm{SS}}\right)$ to a negative value. This is possible by requiring hierarchies in the soft parameters, for a numerical analysis see [14].

${ }^{5}$ This possibility was anticipated in [10] and proposed also in [17].
} 
breaking the scalar potential of the Higgs sector develops soft terms which read

$$
\begin{aligned}
V_{\text {soft }}\left(H_{u}, H_{d}, S\right)= & m_{h_{u}}^{2}\left|H_{u}\right|^{2}+m_{h_{d}}^{2}\left|H_{d}\right|^{2}+m_{3}^{2}\left(H_{u} H_{d}+\text { h.c. }\right) \\
& +m_{h_{s}}^{2}|S|^{2}+\left(y_{s} A_{\lambda} S H_{u} H_{d}+\frac{1}{2} b_{s}^{2} S^{2}+\text { h.c. }\right),
\end{aligned}
$$

where $m_{j}$ are the soft scalar masses, $A_{y}$ the $A$-term and $m_{3}^{2}, b_{s}^{2}$ are the $b$-term and bilinear soft term of the singlet respectively. ${ }^{6}$

The explicit computation of the scalar CP-even Higgs potential shows that the condition, at the scale $M_{\mathrm{SS}}{ }^{7}$

$$
\hat{m}_{3}^{4} \simeq\left(m_{h_{u}}^{2}+\mu^{2}\right)\left(m_{h_{d}}^{2}+\mu^{2}\right)
$$

with $\mu=\mu_{h}+y_{s}\langle S\rangle$ and $\hat{m}_{3}^{2}=m_{3}^{2}+y_{s}\left(A_{\lambda}+\mu_{s}\right)\langle S\rangle$, generates a massless Higgs field given by the combination

$$
H_{\mathrm{SM}}=\sin \beta H_{u}-\cos \beta \epsilon H_{d}^{*}
$$

where $\epsilon$ is the antisymmetric tensor and the angle $\beta$ is determined by

$$
\tan ^{2} \beta=\frac{\left|m_{h_{d}}^{2}+\mu^{2}\right|}{\left|m_{h_{u}}^{2}+\mu^{2}\right|}
$$

with $m_{h_{u}}, m_{h_{d}}$ and $\mu$ evaluated at the scale $M_{\text {SS }}$. It can be easily seen that the choice of superpotential in equation (2.1) yields $\langle S\rangle \ll m_{\mathrm{EW}}$ and thus the singlet contribution to $\mu$ is suppressed and can be neglected. Moreover, this implies effective quadratic terms are subleading and hence the fine-tuning conditions in (2.3) and (2.5) are identical to the MSSM case.

Assuming the generic situation that the susy particles get masses of $\mathcal{O}\left(M_{\mathrm{SS}}\right)$ they can be integrated out, leaving an effective Standard Model description at energies below the cutoff scale $M_{\mathrm{SS}}$. Furthermore, the explicit computation of the effective Lagrangian provides the boundary condition for the quartic coupling in the Standard Model potential given in (3.1). These are the so called matching conditions and at tree level they read

$$
\lambda^{\text {tree }}\left(M_{\mathrm{SS}}\right)=\frac{1}{4}\left(g_{2}^{2}+\frac{3}{5} g_{1}^{2}\right) \cos ^{2} 2 \beta+\frac{1}{2} y_{s}^{2}(1-\delta) \sin ^{2} 2 \beta
$$

where

$$
\delta=\frac{\left(2 \mu_{h} / \sin 2 \beta-A_{\lambda}-\mu_{s}\right)^{2}}{m_{s}^{2}+b_{s}^{2}+\mu_{s}^{2}} .
$$

The first term in (2.6) is the well known D-term contribution in the MSSM while the second term appears only in the singlet-extension. The latter is generated by two effects, an F-term contribution generated by the Yukawa interaction that couples the singlet to the Higgs, and an extra contribution (proportional to $\delta$ ) originated from integrating out the

\footnotetext{
${ }^{6}$ It is worth pointing out that, unless they are forbidden by a symmetry, effective soft cubic and linear terms for the singlet can be generated radiatively. As long as $y_{s}$ is small, the latter are suppressed and can be neglected. Throughout this letter we will not consider them in the calculations.

${ }^{7}$ Here we used that the off-diagonal terms in the mass matrix (of the three CP-even scalars) that mix the MSSM Higgses with the singlet can be neglected in the diagonalization.
} 
singlet. Interestingly, the denominator in (2.7) corresponds to the mass of the (CP-even) scalar singlet and, thus, it is positive. In turn, $\delta$ can only take positive values and the correction to $\lambda\left(M_{\mathrm{SS}}\right)$ is always negative.

The matching given in (2.6) receives higher order threshold corrections $\left(\delta \lambda^{\text {th }}\right)$ that for the MSSM were originally computed at one loop level in [10] (and recently reviewed in [14] with leading two loop effect). We follow [14] and parametrize the corrections as follows ${ }^{8}$

$$
\delta \lambda^{\mathrm{th}}\left(M_{\mathrm{SS}}\right)=\Delta \lambda^{1 l, \mathrm{reg}}+\Delta \lambda^{1 l, \phi}+\Delta \lambda^{1 l, \chi^{1,2}} .
$$

These originate from the change of renormalization schemes that relate the gauge couplings in the $\overline{\mathrm{DR}}$ scheme to the $\overline{\mathrm{MS}}$ scheme $\left(\Delta \lambda^{1 l, \text { reg }}\right)$ and from integrating out the heavy scalars $\left(\Delta \lambda^{1 l \phi}\right)$ and fermionic superpartners $\left(\Delta \lambda^{1 l \chi^{1,2}}\right)$. The effect induced from stop mixing is also included, with the stop mixing parameter defined as

$$
\tilde{X}_{t}=\left(A_{t}-\mu_{h} \cot \beta\right)^{2} /\left(m_{Q} m_{U}\right) .
$$

Let us comment that one loop threshold corrections generated by the stop mixing can, in principle, take negative values. However, these require very large $\tilde{X}_{t}$ for which vacuum stability is severely threaten. An analytic bound upon $\tilde{X}_{t}$ as a function of the stop soft masses was derived in [14] together with a numerical analysis on its effect on the Higgs mass. Furthermore, those values consistent with the bound yield positive corrections to $\lambda\left(M_{\mathrm{SS}}\right)$ and therefore enhance the Higgs mass with respect to the tree level result. Finally, it is worth pointing out that the one loop threshold corrections introduced in $\delta^{\text {th }} \lambda\left(M_{\mathrm{SS}}\right)$ can take either sign. These are negligible for nearly degenerated soft masses, but can be sizeable for lower $M_{\mathrm{SS}}$ if hierarchies among the soft parameters are allowed. ${ }^{9}$

Before finishing this section it is worth noticing that, as long as one does not assume a special pattern of soft terms, the free parameters that determine $\lambda\left(M_{\mathrm{SS}}\right)$ in (2.6) are

$$
y_{s}, \delta, \tan \beta \text { and } M_{\mathrm{SS}} \text {. }
$$

However, if the soft terms are specified, the corresponding soft parameters at $M_{\mathrm{SS}}$ completely determine $\tan \beta$ and $\delta$ via (2.5) and (2.7) respectively.

\section{Calculation of the Higgs mass}

The Standard Model Higgs potential reads

$$
V_{\mathrm{SM}}=\frac{1}{2} \lambda\left(|H|^{2}-v^{2}\right)^{2}
$$

\footnotetext{
${ }^{8}$ In our setup these are not complete, the threshold corrections coming from integrating out the two scalar singlets and the singlino are not included.

${ }^{9}$ For a numerical study of this effect see [14]. In particular, it was shown that varying soft masses within $0.3 M_{\mathrm{SS}}<m_{i}<3 M_{\mathrm{SS}}$ can yield $2 \mathrm{GeV}$ uncertainty on the Higgs mass at $M_{\mathrm{SS}} \simeq 10^{16} \mathrm{GeV}$ and up to $6 \mathrm{GeV}$ for $M_{\mathrm{SS}} \simeq 10^{4} \mathrm{GeV}$.
} 
where $v=\sqrt{2} \cdot 174.1 \mathrm{GeV}$ is the the Higgs vacuum expectation value. ${ }^{10}$ From (3.1) one immediately learns that value the of $\lambda$ determines the Higgs mass $M_{h}$ at loop level via

$$
M_{h}^{2}=v^{2}\left(\lambda+\delta_{\lambda}\right)
$$

It is worth recalling that the top Yukawa coupling $y_{t}$ is fixed at loop level by the mass of the top quark through

$$
y_{t}=\frac{m_{t}}{v} \sqrt{2}\left(1+\delta_{t}\right)
$$

$\delta_{\lambda}$ and $\delta_{t}$ parametrize the threshold corrections at the renormalization scale $\left(m_{t}\right)$ that were originally computed in [18] and [19] respectively. The state-of-the-art computations are given at the two loop level (and dominant three loop correction for $y_{t}$ ) in [5].

\subsection{Matching at $M_{\mathrm{SS}}$}

We perform the numerical calculations using a modified version of SPheno-3.3.6 [20, 21] created by SARAH-4.5.8 [22-24]. Given $\lambda\left(M_{\mathrm{SS}}\right)$ the Renormalization Group Equations (RGEs) are calculated at two-loop-level to yield the couplings at the weak scale. All couplings are renormalized at one loop at $m_{t}$ in the $\overline{\mathrm{MS}}$ scheme and the corresponding Higgs mass is thus calculated at one loop level. For the top Yukawa coupling we include the two loop and dominant three loop QCD correction given in eq. (57) in [5]. For completeness we provide the values of the SM parameters used in the calculations

$$
\begin{aligned}
m_{t} & =173.34 \pm 0.76 \mathrm{GeV} & \alpha_{s} & =0.1184 \\
M_{z} & =91.18 \mathrm{GeV}, & G_{F} & =1.16637 \cdot 10^{-5} .
\end{aligned}
$$

A theoretical uncertainty on the Higgs mass of $3 \mathrm{GeV}$ is generically applied to supersymmetric models. This was computed within the MSSM in [25], assuming low energy values of the SUSY-breaking scale. The computation was recently reviewed in [26], for arbitrary (large) values of the SUSY-breaking scale and yielded a $1 \mathrm{GeV}$ uncertainty for the Higgs mass. In the following we use this result.

\subsection{Matching at $M_{\mathrm{GUT}}$}

In this section we explain how to obtain $\lambda\left(M_{\mathrm{SS}}\right)$ from a set of universal soft terms at the GUT scale, these are specified in (4.1). The procedure to calculate $\lambda\left(M_{\mathrm{SS}}\right)$ goes as follows. The values of the gauge and top Yukawa couplings in the NMSSM, $\hat{g}_{1}, \hat{g}_{2}, \hat{g}_{3}, \hat{y}_{t}$, are fixed by the corresponding $g_{1}, g_{2}, g_{3}, y_{t}$ in the SM via the matching conditions at $M_{\mathrm{SS}} \cdot{ }^{11}$ The matching conditions are given at tree level by

$$
\hat{g}_{1}=\sqrt{\frac{5}{3}} g^{\prime}, \quad \hat{g}_{2}=g_{2}, \quad \hat{g_{3}}=g_{3}, \quad \hat{y}_{t}=\frac{y_{t}}{\sin \beta}
$$

\footnotetext{
${ }^{10}$ In the literature another convention for $v$ is often used, without the squared root of 2 . In that case, $M_{h}^{2}=2 \lambda v^{2}$, the result is of course independent of this definition.

${ }^{11}$ The corresponding values of $\hat{y}_{t}, \hat{g}_{1}, \hat{g}_{2}, \hat{g}_{3}$ used correspond to $m_{h}=125 \mathrm{GeV}$. Variations of $m_{h}$ between 50 and $150 \mathrm{GeV}$ yield variations of $10^{-6}$ in the gauge couplings and $10^{-3}$ in the top Yukawa.
} 
and receive one loop threshold corrections which can be found in [14]. However, gauge couplings only enter in the calculation of the Higgs mass via the RGEs for the soft terms and thus, higher order corrections can be neglected. ${ }^{12}$ With $\hat{y}_{t}, \hat{g}_{1}, \hat{g}_{2}, \hat{g}_{3}$ at hand we run from $M_{\mathrm{SS}}$ up to $M_{\mathrm{GUT}}$, with the RGEs of the NMSSM at two loops, to calculate $\hat{y}_{t_{0}}$, $\hat{g}_{1_{0}}, \hat{g}_{2_{0}}, \hat{g}_{3_{0}}$. Using the latter boundary conditions for gauge and top Yukawa couplings together with (4.1) we implement the RGEs of the NMSSM again at the two loop level for Yukawa and gauge couplings and at one loop level for the soft parameters, to obtain the corresponding soft terms at $M_{\mathrm{SS}}$. The RGEs are given in [16] and we neglect the contribution of all Yukawa couplings except $\hat{y}_{t} \cdot{ }^{13}$ The soft parameters at $M_{\mathrm{SS}}$ determine the values of $\tan \beta$ and $\delta$ via the eqs. (2.5) and (2.7) respectively which yield $\lambda\left(M_{\mathrm{SS}}\right)$ through (2.6). ${ }^{14}$ The one loop corrections given in (2.8), using the stop mixing in (2.9) are also included. With the value of $\lambda\left(M_{\mathrm{SS}}\right)$ at hand, we proceed as in 3.1.

\section{The Higgs mass as a function of $M_{\mathrm{SS}}$}

In this section we study the Higgs mass as a function of $M_{\mathrm{SS}}$, varying the NMSSM parameters (2.10) that determine the value of $\lambda\left(M_{\mathrm{SS}}\right)$ in (2.6). In section 4.3 we proceed as before but using the values of $\tan \beta$ and $\delta$ in $\lambda\left(M_{\mathrm{SS}}\right)$ computed from universal patterns of soft terms at the GUT scale.

\section{1 $\lambda>0$ at SUSY-breaking scale}

To begin with, notice that the MSSM-like contribution to (2.6) vanishes for $\tan \beta$ equal to one, while the NMSSM piece is maximized. From this observation we learn that in the NMSSM low values of $\tan \beta$ can severely lessen the upper bound of $M_{\mathrm{SS}}$ as $y_{s}$ increases. In figure 1 and 2 we plot the Higgs mass as a function of $M_{\mathrm{SS}}$ for different values of $y_{s}$, assuming $\delta=0$ and $\tan \beta=1,2$ respectively. For large $\tan \beta$ the situation resembles the MSSM case $[10,11]$. In this regime the first term in (2.6) is maximized and thus pulls $M_{\mathrm{SS}}$ to low energies while the NMSSM term is almost vanishing regardless of the value of $y_{s}$.

\section{$4.2 \lambda<0$ at SUSY-breaking scale}

As can be seen from (2.6) by setting $\delta=0$, all universal contributions are strictly positive. This implies that independently of the values of the parameters in the supersymmetric theory, there exists a strict upper bound on $M_{\mathrm{SS}}$ (within uncertainties) fixed by the scale $\mu_{0}$ at which $\lambda\left(\mu_{0}\right)$ vanishes, equivalently, the scale at which the SM becomes unstable. The state-of-the-art computation yields $\mu_{0} \simeq 10^{10 \pm 1} \mathrm{GeV}$ [5]. However, if $\lambda\left(M_{\mathrm{SS}}\right)$ can be matched to the expected negative value determined by the running of $\lambda$ in the SM, the upper bound on $M_{\mathrm{SS}}$ disappears. Let us remark that this statement assumes the metastability of the Standard Model vacuum is not spoiled by the UV-completion. The metastability bound relies on the top and Higgs masses, for a precise determination of this

\footnotetext{
${ }^{12}$ These could become important in precise estimations of gauge coupling unification.

${ }^{13}$ Neglecting the bottom Yukawa is a good approximation for low (or moderate) values of $\tan \beta$.

${ }^{14}$ Notice that the value of $\tan \beta$ already appears in the matching condition of the top Yukawa given in (3.5), the values should of course match.
} 


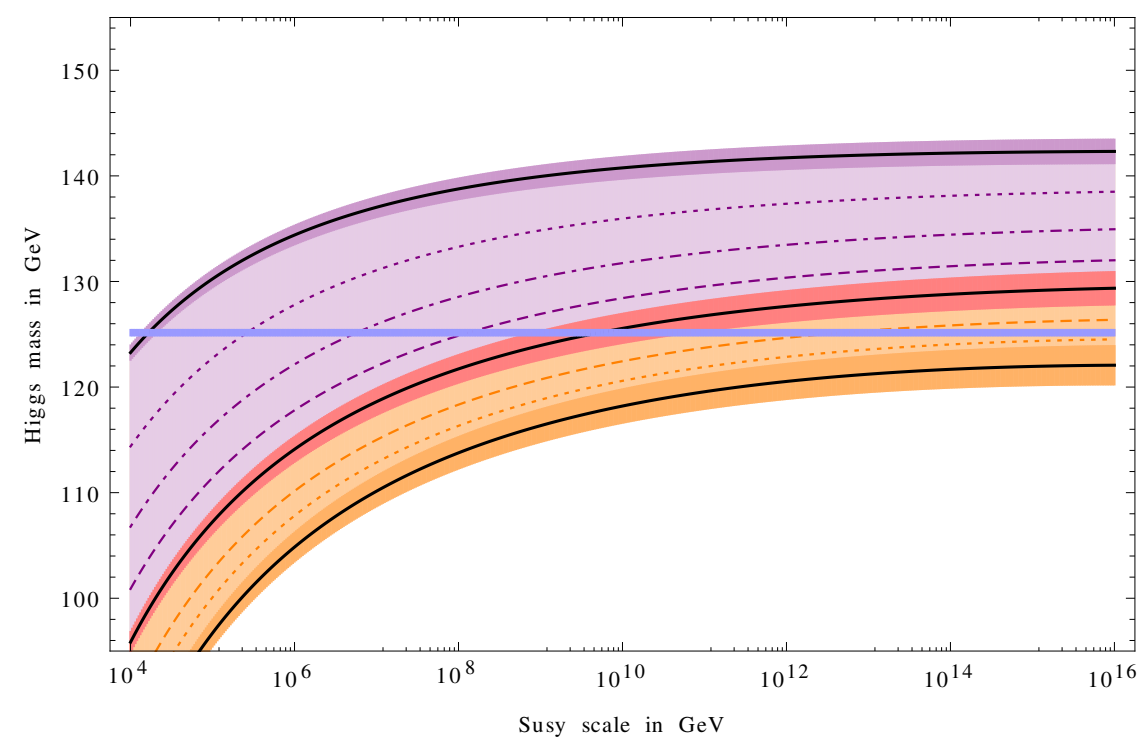

Figure 1. Higgs mass as a function of $M_{\mathrm{SS}}$ for $\tan \beta=1$. The region shaded in violet(orange) corresponds to $\lambda>0(\lambda<0)$ and from bottom-up $y_{s}=0.2,0.3,0.4,0.5\left(y_{s}=0.3,0.25,0.2\right.$ with $\delta=-2)$. In red $\lambda=0, y_{s}=0$. We assumed $\tilde{X}_{t}=0$ and degenerate superparticles at $M_{\mathrm{SS}}$. The bands display the uncertainty from varying $m_{t}=173.34 \pm 0.76 \mathrm{GeV}$, we did not include them in all the curves to avoid clutter. The line in blue is the measured Higgs mass $125.15 \pm 0.25 \mathrm{GeV}$.

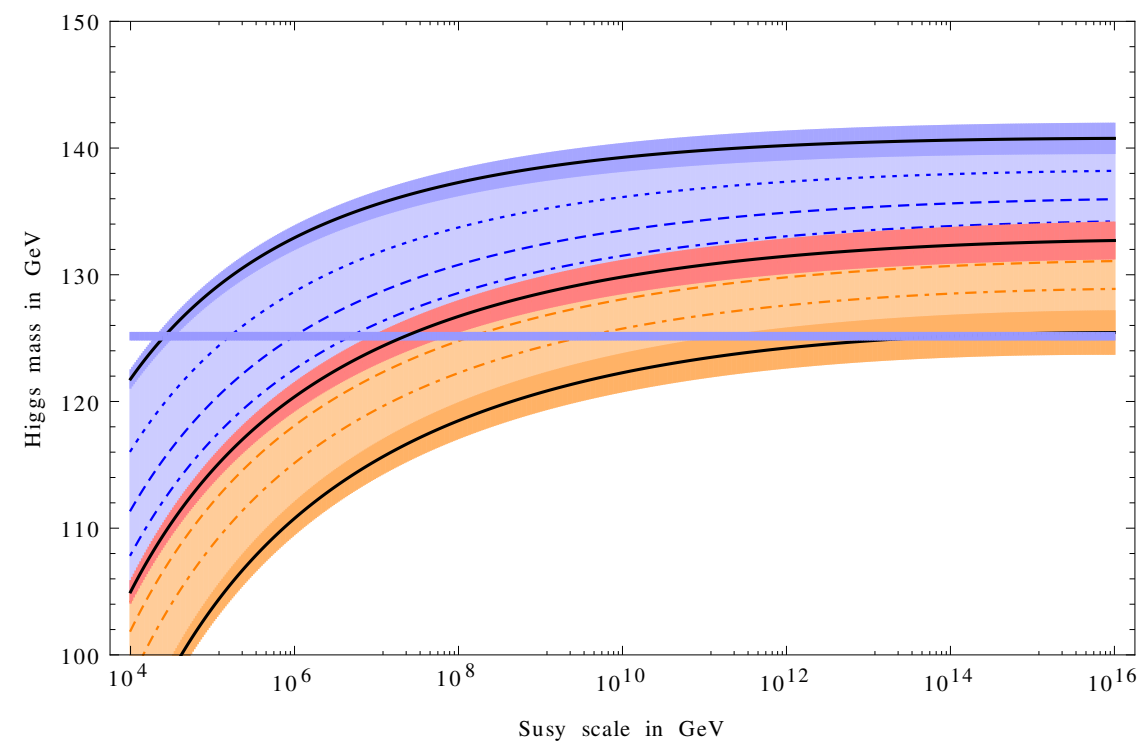

Figure 2. Higgs mass as a function of $M_{\mathrm{SS}}$ for $\tan \beta=2$. The region shaded in blue(orange) corresponds to, from bottom-up, $y_{s}=0.2,0.3,0.4,0.5\left(y_{s}=0.4,0.3,0.2\right.$ with $\left.\delta=-2\right)$. In red $y_{s}=0$. We assumed $\tilde{X}_{t}=0$ and degenerate superparticles at $M_{\mathrm{SS}}$. The bands display the uncertainty from varying $m_{t}=173.34 \pm 0.76 \mathrm{GeV}$. The line in blue is the measured Higgs mass $125.15 \pm 0.25 \mathrm{GeV}$. 
region see $[5,8]$. Thus, as long as these agree with the experimentally measured values, $M_{\mathrm{SS}}$ can lie at any scale between $\mu_{0}$ and $M_{\mathrm{GUT}}{ }^{15}$ In figures 1 and 2 we plot the Higgs mass for various $y_{s}$, using $\delta=-2$ and $\tan \beta=1,2$ respectively. In these examples it can be clearly seen that $M_{\mathrm{SS}}$ could take values up to $M_{\mathrm{GUT}}$.

\subsection{Universal soft terms at $M_{\mathrm{GUT}}$}

As already anticipated, in this section we study the Higgs mass as a function of $M_{\mathrm{SS}}$ assuming an underlying structure of universal soft terms at the GUT scale. In the NMSSM these are specified by the following parameters

$$
m_{0}, M_{0}, A_{0}, \mu_{h_{0}}, \mu_{s_{0}} \text { and } b_{s_{0}}
$$

where $M_{0}$ denote the gaugino masses and the remaining soft terms are as introduced in section $2 .{ }^{16}$ The subindex 0 indicates that the values are defined at the GUT scale, with $M_{\mathrm{GUT}}=10^{16} \mathrm{GeV}$. Examples of universal soft terms in string theory can be obtained e.g. in the dilaton domination scenario [31].

Notice from (2.5) that $\tan \beta$ is equal to one at the GUT scale and as $m_{h_{u}}^{2}, m_{h_{d}}^{2}$ and $\mu_{h}$ run, $\tan \beta$ evolves accordingly. However, $\tan \beta$ remains close to one for large values of $M_{\text {SS }}$. In other words, the unification (or universality) of soft masses predicts small values of $\tan \beta$, as already pointed out in [32]. For low values of the SUSY-breaking scale, $m_{h_{u}}^{2}$ becomes smaller and eventually changes sign, at this point there is a sudden increase of $\tan \beta$.

On the other hand, $m_{s}, b_{s}$ and $\mu_{s}$ appearing in $\delta$ do not run for small values of $y_{s}$ and stay equal to their boundary conditions at the GUT scale. Thus, the running of $\delta$ is induced by $\mu_{h}$ and $A_{\lambda}$. As long as there are no hierarchies among the couplings, $\delta$ stays constant at large $M_{\mathrm{SS}}$ and increases for low values of the SUSY-breaking scale.

In sum, two effects determine the Higgs mass in this scheme. At large values of $M_{\mathrm{SS}}$, $\tan \beta$ takes very small values and so enhances the NMSSM correction to $\lambda\left(M_{\mathrm{SS}}\right)$. In this regime, $\delta$ does not significantly vary and, thus, the Higgs mass dependence upon $M_{\mathrm{SS}}$ is (almost) constant. Furthermore, by tuning the value of $y_{s}$ near $\mathcal{O}\left(10^{-2}\right)$, the Higgs mass can be easily accommodated in the experimental bound. For lower values of $M_{\mathrm{SS}}, \tan \beta$ starts to increase and thus $\lambda\left(M_{\mathrm{SS}}\right)$ becomes insensitive to the NMSSM correction. The latter competes with the MSSM contribution which grows with $\tan \beta$. The sum of these two terms leads to a slow decrease of the Higgs mass with $M_{\mathrm{SS}}$.

In figure 3 we show the Higgs mass as a function of $M_{\mathrm{SS}}$ for the following choice of soft masses at the GUT scale, i.e. $m_{0}=M_{0}=\mu_{s_{0}}, b_{s_{0}}^{2}=-\mu_{s}^{2}$ and $A_{0}=\mu_{h_{0}}=-1.5 M_{0}$, and various $y_{s} \cdot{ }^{17}$ As $y_{s}$ decreases the upper bound on $M_{\mathrm{SS}}$ approaches the lower bound of $M_{\mathrm{SS}} \simeq 10^{10} \mathrm{GeV}$, that corresponds to the limit of $\tan \beta=1$ and $y_{s}=0$. Whereas increasing $y_{s}$ enhances the NMSSM negative contribution to $\lambda\left(M_{\mathrm{SS}}\right)$ and, thus, pushes $M_{\mathrm{SS}}$ to larger

\footnotetext{
${ }^{15}$ One should observe, however, that higher order operators encoding possible effects of new physics could modify the behavior of the quartic coupling near the matching scale and turn it to the unstable regime, spoiling the metastability of the SM vacuum [27] (see also [28-30]).

${ }^{16}$ Notice that $m_{3}$ is fixed by the fine-tuning condition (2.3).

${ }^{17}$ Notice that this choice of $A_{0}$ and $\mu_{h_{0}}$ minimizes the effect of stop mixing at large SUSY scales.
} 


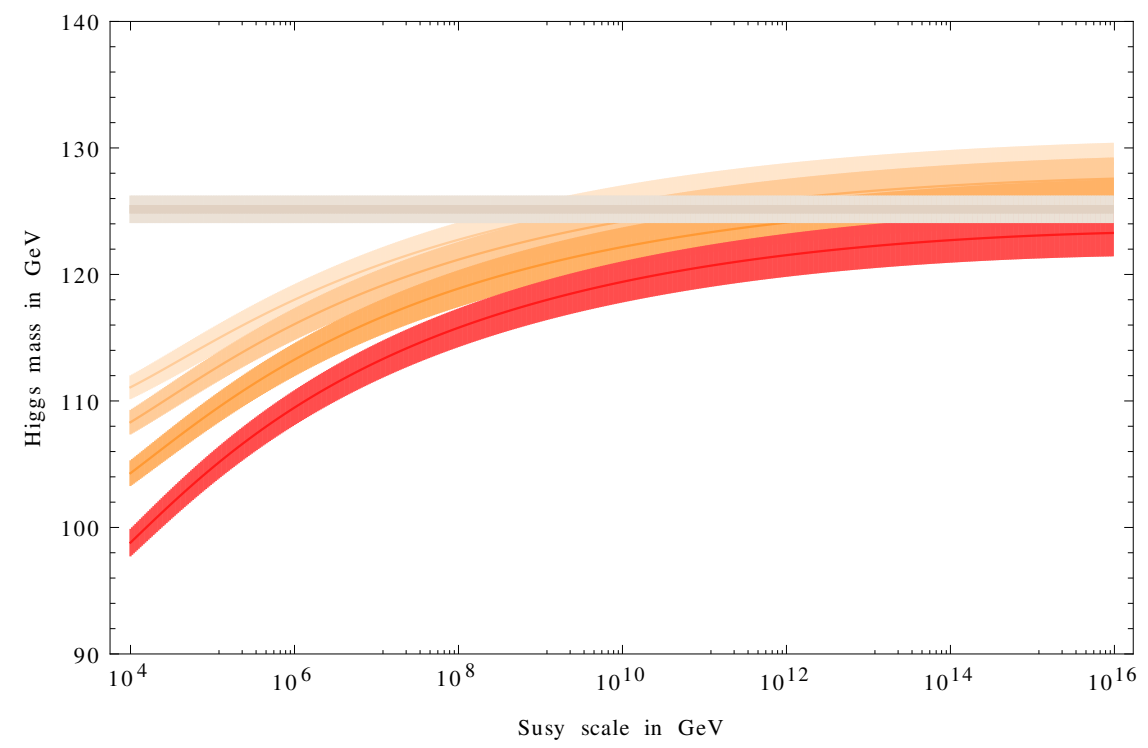

Figure 3. Higgs mass as a function of $M_{\mathrm{SS}}$ with soft terms at the GUT scale: $m_{0}=M_{0}=\mu_{s_{0}}$, $b_{s_{0}}^{2}=-\mu_{s_{0}}^{2}$ and $A_{0}=\mu_{h_{0}}=-1.5 M_{0}$ and $y_{s}=0.05$ (beige), 0.075 (light orange), 0.1 (dark orange), 0.125 (red). The bands correspond to the uncertainty bound in $m_{t}$ and the region in lighter brown is $m_{h}=125 \pm 1 \mathrm{GeV}$. In darker brown the experimental bound on $m_{h}$.
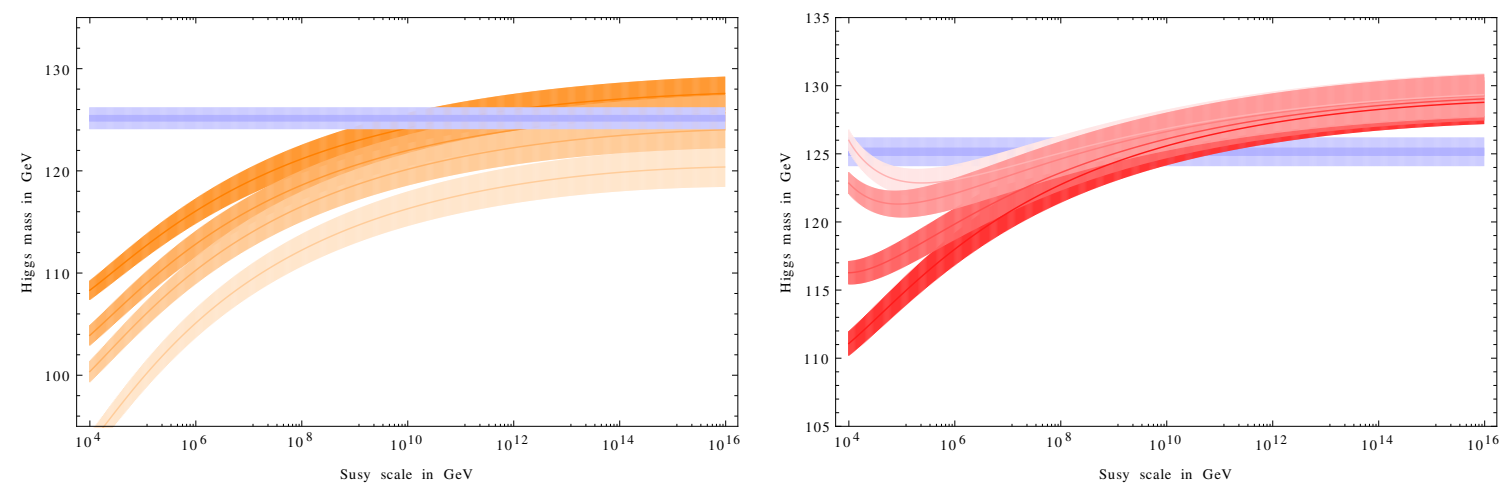

Figure 4. Higgs mass as a function of $M_{\mathrm{SS}}$. The bands correspond to the uncertainty bound in $m_{t}$ and the region in blue is $m_{h}=125 \pm 1 \mathrm{GeV}$. In darker blue the experimental bound on $m_{h}$. (Left) $y_{s}=0.075$ and (from dark to light orange) $m_{0}^{2}=M_{0}^{2}, 0.6 M_{0}^{2}, 0.45 M_{0}^{2}, 0.3 M_{0}^{2}$. (Right) $y_{s}=0.05$ and (from dark to light red) $\mu_{h_{0}}=-1.5 M_{0},-1.25 M_{0},-M_{0},-0.9 M_{0}$.

values. Similarly, in figure 4 (left), by fixing $y_{s}$ we show the effect of lowering $\lambda\left(M_{\mathrm{SS}}\right)$ by incrementing $\delta$. This can be achieved by taking smaller values for the soft masses.

Notice that in the limit of vanishing $y_{s}$, the NMSSM contribution to $\lambda\left(M_{\mathrm{SS}}\right)$ is negligible. Hence, larger $\tan \beta$ values raise the Higgs mass at low energies. This effect is manifest depending on the choice of soft terms, in particular of $\mu_{h_{0}}{ }^{18}$ In figure 4 (right) we show the Higgs mass as a function of $M_{\mathrm{SS}}$ for fixed $y_{s}$ and the same soft terms as before but varying $\mu_{h_{0}}$. As seen in figure 4 , for lower $\mu_{h_{0}}, \tan \beta$ becomes larger and boosts the Higgs mass.

\footnotetext{
${ }^{18}$ The choice of soft masses and A-terms, i.e. $m_{0}$ and $A_{0}$ have a milder effect on $\tan \beta$.
} 


\section{Conclusions}

In this letter we studied the Higgs mass in the NMSSM within High-Scale Supersymmetry. In this setup, the low energy effective theory is described by the Standard Model and the respective couplings, in particular the quartic coupling $\lambda$, are fixed by the supersymmetric theory at the SUSY-breaking scale $M_{\mathrm{SS}}$. In the MSSM this matching sets $\lambda\left(M_{\mathrm{SS}}\right)$ to a positive (or vanishing) value and, thus, sets an upper bound $M_{\mathrm{SS}} \lesssim 10^{10} \mathrm{GeV}$. In the NMSSM, $\lambda\left(M_{\mathrm{SS}}\right)$ receives additional tree level corrections given in (2.6). The latter allow $\lambda\left(M_{\mathrm{SS}}\right)$ to take negative values, and, thus, the SUSY-breaking scale can take any value up to the GUT scale. In this work, we computed the Higgs mass as a function of the SUSYbreaking scale varying the NMSSM parameters. The results are summarized in figures 1 and 2. For large values of $M_{\mathrm{SS}}$, the Higgs mass becomes almost constant and can be easily adjusted to the experimental bound $125.15 \pm 0.25 \mathrm{GeV}$.

In addition, we studied special scenarios of supersymmetry breaking. In particular, we assumed universal soft terms at the GUT scale to constrain the NMSSM parameters appearing in $\lambda\left(M_{\mathrm{SS}}\right)$. The unification of masses yields low values of $\tan \beta$ at large $M_{\mathrm{SS}}$ and, thus, enhances the NMSSM negative contribution to $\lambda\left(M_{\mathrm{SS}}\right)$. For

$$
10^{9} \mathrm{GeV} \lesssim M_{\mathrm{SS}} \lesssim 10^{16} \mathrm{GeV}
$$

the Higgs mass stays almost constant, and its value can be easily accomodated within the experimental bound. ${ }^{19}$ For lower values of $M_{\mathrm{SS}}$ consistency with the Higgs mass becomes more model dependent and requires small NMSSM contributions to $\lambda\left(M_{\mathrm{SS}}\right) .{ }^{20}$ Furthermore, in this regime the soft masses spread out and, thus, one loop contributions to $\lambda\left(M_{\mathrm{SS}}\right)$ become large. Hence, for low values of $M_{\mathrm{SS}}$ the determination of the Higgs mass relies on the details of the soft parameters.

Let us remark that although these scenarios suffer from a severe hierarchy problem, introduced by the fine-tuning of the electroweak scale, supersymmetry might nevertheless be present at high scales as required by UV-completions e.g. string theory. In this case, it is conceivable that intermediate supersymmetric theories appear at scales between $M_{\mathrm{SS}}$ and a given cutoff $\Lambda$, e.g. the string or Planck scale. As long as $M_{\mathrm{SS}} \ll \Lambda$, non-renormalizable couplings between hidden fields and the NMSSM fields can introduce corrections to the matching conditions together with higher dimensional operators in the effective Standard Model potential which are suppressed and can be safely neglected. ${ }^{21}$

To conclude, even if not directly accessible at the LHC, consistency with the Higgs mass via the matching conditions is a necessary condition for models with large SUSY-breaking scales. As we have studied in this letter, the NMSSM can support SUSY-breaking scales

\footnotetext{
${ }^{19}$ See section 4.3 for details.

${ }^{20}$ In particular, $\tan \beta$ should take larger values and $y_{s}$ should be negligible in order to suppress the singlet contribution to $\lambda\left(M_{\mathrm{SS}}\right)$.

${ }^{21}$ Here we denote as hidden fields unknown fields whose dynamics are relevant at scales within $M_{\mathrm{SS}}$ and $\Lambda$. Note that if the hidden field is responsible for supersymmetry breaking, soft terms are generated. These were already included in the Lagrangian and they are assumed to sit at a scale $M_{\mathrm{SS}}$. If the hidden field couples via renormalizable operators to the NMSSM fields then the matching should be computed to a different theory and it would not make sense to talk about the NMSSM any longer.
} 
near the GUT scale. These news are of particular interest for string phenomenology, since generic vacua in the landscape allow for supersymmetry breaking only at high scales. It might be interesting to find explicit examples of the NMSSM from string derived setups. In particular, provided the UV completion yields specific patterns of soft terms, the measured value of the Higgs mass can be used to test whether these are plausible embeddings of the Standard Model or to constrain the parameters of the theory, in particular, $M_{\mathrm{SS}}$.

\section{Acknowledgments}

I would specially like to thank Emanuele Bagnaschi, David Ciupke, Florian Staub, Alexander Voigt and Georg Weiglein for useful discussions. I would also like to thank Wilfried Buchmüller, Jan Louis, Stefano di Vita and Alexander Westphal for interesting comments on the letter. This work is supported by the German Science Foundation (DFG) within the Collaborative Research (CRC) 676 "Particles, Strings and the Early Universe".

Open Access. This article is distributed under the terms of the Creative Commons Attribution License (CC-BY 4.0), which permits any use, distribution and reproduction in any medium, provided the original author(s) and source are credited.

\section{References}

[1] CMS collaboration, Measurement of the properties of a Higgs boson in the four-lepton final state, Phys. Rev. D 89 (2014) 092007 [arXiv:1312.5353] [InSPIRE].

[2] CMS collaboration, Observation of the diphoton decay of the Higgs boson and measurement of its properties, Eur. Phys. J. C 74 (2014) 3076 [arXiv: 1407.0558] [INSPIRE].

[3] ATLAS collaboration, Measurement of the Higgs boson mass from the $H \rightarrow \gamma \gamma$ and $H \rightarrow Z Z^{*} \rightarrow 4 \ell$ channels with the ATLAS detector using $25 \mathrm{fb}^{-1}$ of pp collision data, Phys. Rev. D 90 (2014) 052004 [arXiv: 1406.3827] [InSPIRE].

[4] M.R. Douglas and S. Kachru, Flux compactification, Rev. Mod. Phys. 79 (2007) 733 [hep-th/0610102] [INSPIRE].

[5] D. Buttazzo et al., Investigating the near-criticality of the Higgs boson, JHEP 12 (2013) 089 [arXiv: 1307.3536] [INSPIRE].

[6] J. Ellis, J.R. Espinosa, G.F. Giudice, A. Hoecker and A. Riotto, The probable fate of the standard model, Phys. Lett. B 679 (2009) 369 [arXiv:0906.0954] [inSPIRE].

[7] J. Elias-Miró et al., Higgs mass implications on the stability of the electroweak vacuum, Phys. Lett. B 709 (2012) 222 [arXiv:1112.3022] [INSPIRE].

[8] G. Degrassi et al., Higgs mass and vacuum stability in the standard model at NNLO, JHEP 08 (2012) 098 [arXiv: 1205.6497] [INSPIRE].

[9] L.J. Hall and Y. Nomura, A finely-predicted Higgs boson mass from a finely-tuned weak scale, JHEP 03 (2010) 076 [arXiv:0910.2235] [INSPIRE].

[10] G.F. Giudice and A. Strumia, Probing high-scale and split supersymmetry with Higgs mass measurements, Nucl. Phys. B 858 (2012) 63 [arXiv:1108.6077] [InSPIRE].

[11] M.E. Cabrera, J.A. Casas and A. Delgado, Upper bounds on superpartner masses from upper bounds on the Higgs boson mass, Phys. Rev. Lett. 108 (2012) 021802 [arXiv:1108.3867] [INSPIRE]. 
[12] A. Arbey, M. Battaglia, A. Djouadi, F. Mahmoudi and J. Quevillon, Implications of a 125 GeV Higgs for supersymmetric models, Phys. Lett. B 708 (2012) 162 [arXiv:1112.3028] [INSPIRE].

[13] P. Draper, G. Lee and C.E.M. Wagner, Precise estimates of the Higgs mass in heavy supersymmetry, Phys. Rev. D 89 (2014) 055023 [arXiv:1312.5743] [InSPIRE].

[14] E. Bagnaschi, G.F. Giudice, P. Slavich and A. Strumia, Higgs mass and unnatural supersymmetry, JHEP 09 (2014) 092 [arXiv: 1407.4081] [INSPIRE].

[15] A. Delgado, M. Garcia and M. Quirós, Electroweak and supersymmetry breaking from the Higgs boson discovery, Phys. Rev. D 90 (2014) 015016 [arXiv: 1312.3235] [INSPIRE].

[16] U. Ellwanger, C. Hugonie and A.M. Teixeira, The next-to-minimal supersymmetric standard model, Phys. Rept. 496 (2010) 1 [arXiv:0910.1785] [inSPIRE].

[17] A. Hebecker, A.K. Knochel and T. Weigand, The Higgs mass from a string-theoretic perspective, Nucl. Phys. B $\mathbf{8 7 4}$ (2013) 1 [arXiv:1304.2767] [INSPIRE].

[18] A. Sirlin and R. Zucchini, Dependence of the Higgs coupling $h M S(M)$ on $m H$ and the possible onset of new physics, Nucl. Phys. B 266 (1986) 389 [INSPIRE].

[19] K.G. Chetyrkin and M. Steinhauser, The relation between the $\overline{\mathrm{MS}}$ and the on-shell quark mass at order $\alpha_{s}^{3}$, Nucl. Phys. B 573 (2000) 617 [hep-ph/9911434] [InSPIRE].

[20] W. Porod, SPheno, a program for calculating supersymmetric spectra, SUSY particle decays and SUSY particle production at $e^{+} e^{-}$colliders, Comput. Phys. Commun. 153 (2003) 275 [hep-ph/0301101] [INSPIRE].

[21] W. Porod and F. Staub, SPheno 3.1: extensions including flavour, CP-phases and models beyond the MSSM, Comput. Phys. Commun. 183 (2012) 2458 [arXiv:1104.1573] [INSPIRE].

[22] F. Staub, SARAH, arXiv:0806.0538 [inSPIRE].

[23] F. Staub, Automatic calculation of supersymmetric renormalization group equations and self energies, Comput. Phys. Commun. 182 (2011) 808 [arXiv: 1002.0840] [INSPIRE].

[24] F. Staub, SARAH 4: a tool for (not only SUSY) model builders, Comput. Phys. Commun. 185 (2014) 1773 [arXiv:1309.7223] [INSPIRE].

[25] G. Degrassi, S. Heinemeyer, W. Hollik, P. Slavich and G. Weiglein, Towards high precision predictions for the MSSM Higgs sector, Eur. Phys. J. C 28 (2003) 133 [hep-ph/0212020] [INSPIRE].

[26] J. Pardo Vega and G. Villadoro, SusyHD: Higgs mass determination in supersymmetry, JHEP 07 (2015) 159 [arXiv: 1504.05200] [INSPIRE].

[27] L. Di Luzio, G. Isidori and G. Ridolfi, Stability of the electroweak ground state in the standard model and its extensions, Phys. Lett. B 753 (2016) 150 [arXiv:1509.05028] [INSPIRE].

[28] V. Branchina and E. Messina, Stability, Higgs boson mass and new physics, Phys. Rev. Lett. 111 (2013) 241801 [arXiv:1307.5193] [INSPIRE].

[29] V. Branchina, E. Messina and A. Platania, Top mass determination, Higgs inflation and vacuum stability, JHEP 09 (2014) 182 [arXiv:1407.4112] [INSPIRE].

[30] V. Branchina, E. Messina and M. Sher, Lifetime of the electroweak vacuum and sensitivity to Planck scale physics, Phys. Rev. D 91 (2015) 013003 [arXiv:1408.5302] [INSPIRE].

[31] J. Louis, K. Schmidt-Hoberg and L. Zarate, Dilaton domination in the MSSM and its singlet extensions, Phys. Lett. B 735 (2014) 1 [arXiv:1402.2977] [INSPIRE].

[32] L.E. Ibáñez and I. Valenzuela, The Higgs mass as a signature of heavy SUSY, JHEP 05 (2013) 064 [arXiv: 1301.5167] [INSPIRE]. 